\title{
OPTICAL MEASUREMENTS OF THE BEAM PARAMETERS OF THE VEPP-4M STORAGE RING
}

\author{
E. I. Zinin ${ }^{\dagger}$, S. E. Karnaev, V. A. Kiselev, O. I. Meshkov ${ }^{\dagger \dagger}$, N. Y. Mutchnoi, \\ A. N. Selivanov, M. G. Fedotov, BINP, Novosibirsk, Russia
}

\section{Abstract}

Optical diagnostics of the VEPP-4M electron-positron collider are described. The diagnostics provide the data about position and dimensions of the $\mathrm{e}^{-} / \mathrm{e}^{+}$beams. The dissectors are applied for the measurements of the length and the transversal size of the bunches and for studying the collective effects. The linear CCD array provides the by two linear segments. The total circumference of the facility is $366 \mathrm{~m}$. The particles acceleration and compensation of synchrotron losses are provided by 5 resonators that operate at 222 harmonic of the circulation frequency $\mathrm{f}=0.819 \mathrm{MHz}$. Computed dimensions of the beam are: $\sigma_{z}=0.1 \mathrm{MM}, \sigma_{r}=1.5 \mathrm{MM}, \sigma_{\varphi}=5 \mathrm{cM}$ $\left(\mathrm{E}=5 \mathrm{GeV}, \mathrm{U}_{\mathrm{r}}=5 \mathrm{MeV}\right)$. The current physical program

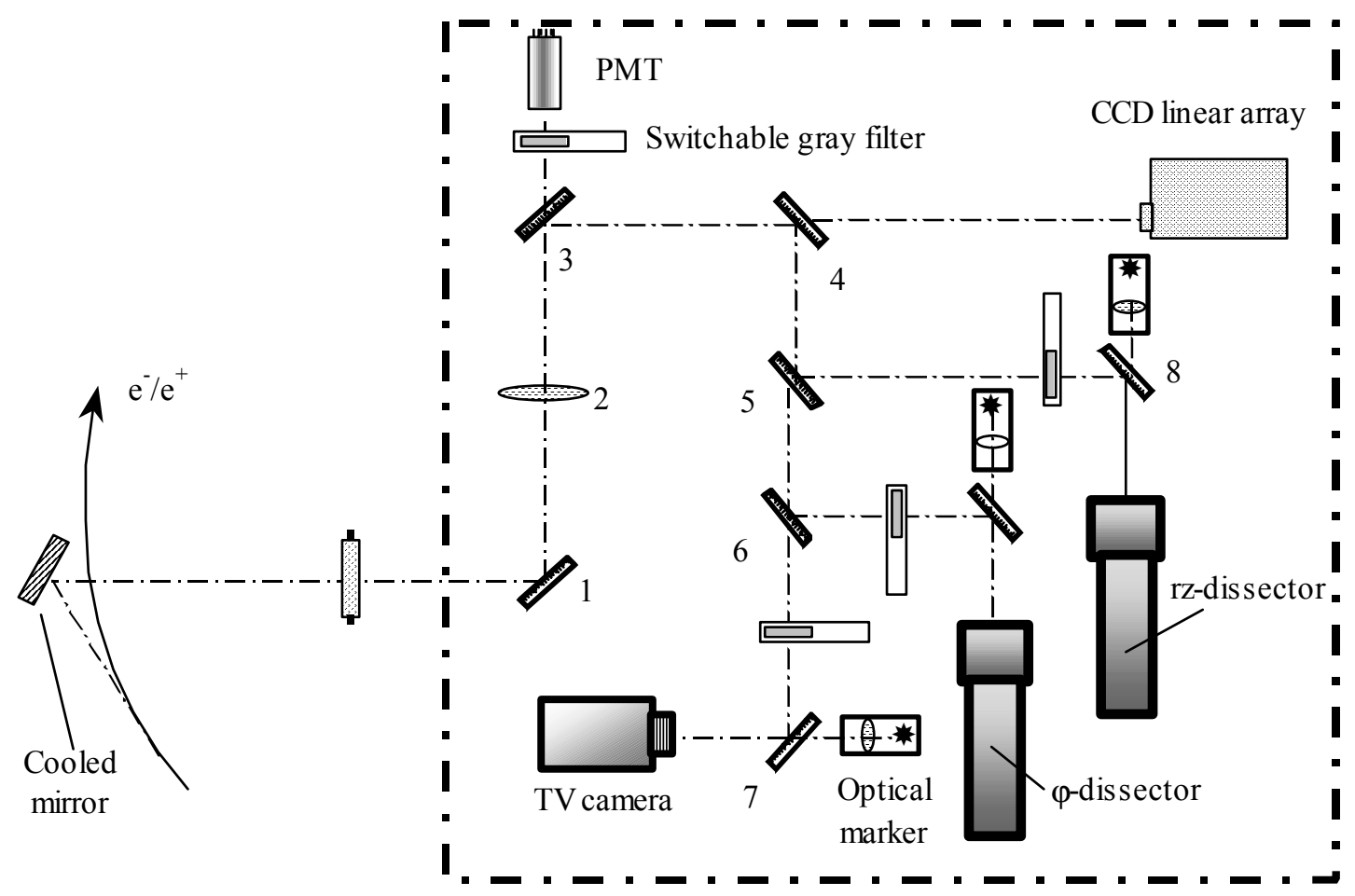

Figure 1: Layout of optical diagnostic for measurement of the $\mathrm{e}^{-} / \mathrm{e}^{+}$beams parameters.

measurements of the vertical size of the beams. The plans of further development of diagnostics are discussed.

\section{INTRODUCTION}

VEPP-4M [1] is an electron-positron collider at the energy up to $6 \mathrm{GeV}$. The collider consists of 2 semi-rings with the average radius $R$ of $45.5 \mathrm{~m}$ which are connected

\footnotetext{
†E.I.Zinin@inp.nsk.su

† O.I.Meshkov@inp.nsk.su
}

of the facility is directed at the energy area of $J / \psi$ meson and $\tau$ lepton (up to $2 \times 2 \mathrm{GeV}$ ). The collider operates under a 2-bunch mode for increasing of the luminosity. The bunches of electrons and positrons are placed at opposite separatrixes.

The optical system for measuring of the beam parameters provides information about beams dimensions, controls the relationship of bunch currents and is employed for studying coherent oscillations of the beams. The optical component of synchrotron radiation (SR) of the beams is used for this. 


\section{LAYOUT OF THE OPTICAL SYSTEM}

Two channels of SR output are located in the bending magnets of the semi-rings and separated by a linear segment. Each channel outputs a radiation of electron or positron beam only. Each channel is equipped with scale of the diagnostic. A remote controlled switchable gray filter expands the dynamic range of the system.

A part of a light passing through the semi-transparent mirror 5 is used for measurement of a longitudinal size of the beam. The dissector with electrostatic focusing and

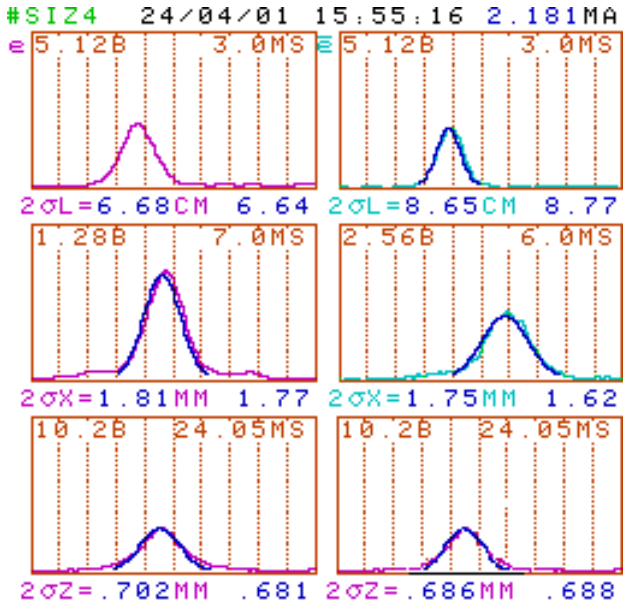

Figure 2: A screen-shot of the computer monitor with the data of the beams dimensions.

practically identical diagnostic systems (fig. 1).

The optical component of SR is reflected from cooled metallic mirror and sent out from the vacuum chamber through a glass window. Mirror 1 matches the SR ray with an optical axis of the system. The beam image is set up on lens 2. Mirrors 3,4,5,8 form a channel for measurement of transverse beam dimensions. The

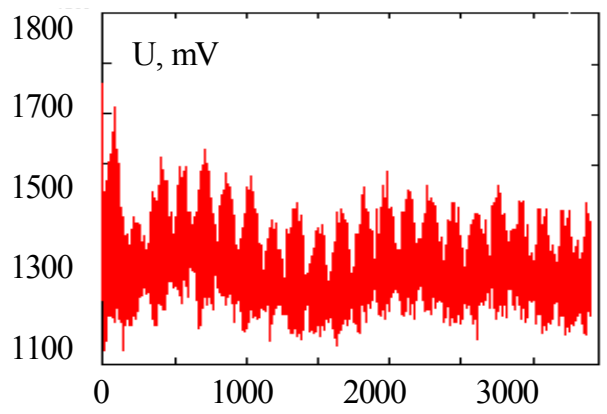

Figure 4a: Investigation of coherent beam-beam effects. The turn-to turn signals, recorded of rz-dissector are displayed. A low-frequency envelope is connected with a tune shift.

dissector with electromagnetic focusing and deflection is used for $\mathrm{r}, \mathrm{z}$ beam dimension measurements (rzdissector). It is equipped with an indexing mechanism for this. The optical marker can project an image of calibrated net to a photo-cathode of the dissector. This device is used for determining and controlling the linear

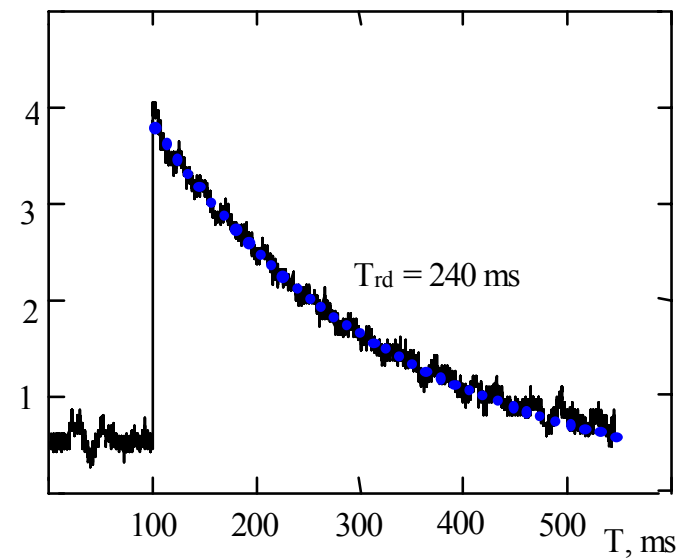

Figure 3: The measurement of radiation dumping time by application of the rz-diss ector.

deflection is employed for this ( $\varphi$-dissector). It has FWHM of instrumental function at $40 \mathrm{ps}$.

The light that passed through the semi-transparent mirror 4 is focused on CCD linear array, which measures $z$ (vertical) size of the beam.

PMT records a light that passed through semitransparent mirror 3. It is applied for precision

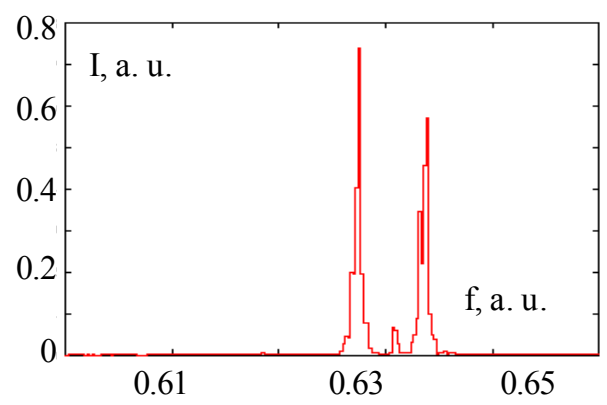

Figure 4b: Fourie spectrum of a signal, presented at fig. $4 \mathrm{a}$..

comparison of the currents of the two electron bunches. This diagnostic is necessary for experiments of energy measurements by the resonant depolarization method [2].

The data of the beam dimensions are displayed at intervals every few seconds on a computer monitor in a control room (fig. 2). Prompt qualitative control of the 
beams position and shape is performed from the TV picture. All the diagnostics that are mentioned above are collected on the common optical plate.

\section{ACCURACY OF BEAM DIMENSIONS MEASUREMENT}

Accuracy of transversal beam dimensions is restricted of angular divergence of SR at the wavelength of the measurement [3]:

$\sigma_{r, z}(\mathrm{~mm}) \approx 0.3 \cdot 10^{-4} \lambda^{2 / 3}(\AA) \cdot R^{1 / 3}(\mathrm{~m}) \approx 0.03 \mathrm{~mm}$. The length of the bunch can be determined to an accuracy of 3 $\mathrm{mm}$ [4]. Comparison of the bunch current is accurate within one percent that is defined by the ADC resolution.

\section{STUDIES ON COLLECTIVE EFFECTS.}

Scanning of the beam image inside rz-dissector is realized across $0.06 \mathrm{~mm}$-slit. This size is much less than the dimension of the beam on a photocatode of the dissector. This allows applying of the device for investigation of the beam oscillations, both at $r$ and $z$ directions. For example, a time of dumping of transversal oscillations can be measured after applying a fast electrostatic impact to the beam or after fast shut down of

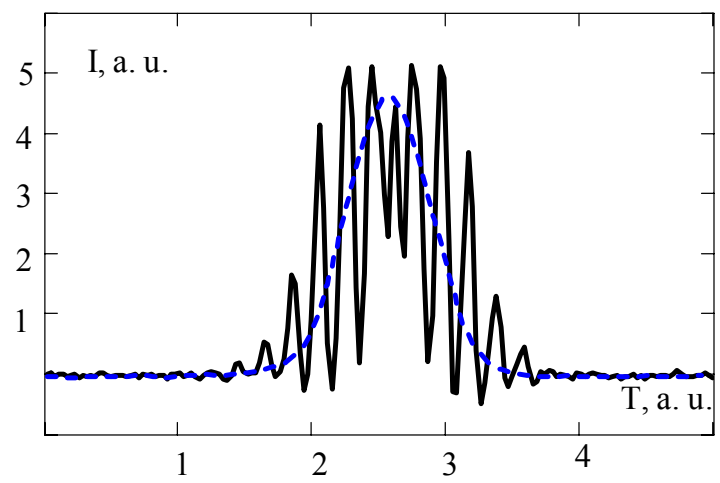

Figure 5: Phase oscillations (solid line) and the unperturbed beam profile (dashed line) recorded of $\varphi$-dissector

a noise excitation of the oscillations (fig. 3). The same method can be applied for investigation of coherent beam-beam effects (fig. 4a, b). There are extensive possibilities for applications of rz- and $\varphi$-dissector for research of the collective effects [3]. There could be mentioned a few applications of this technique: investigations of the beam dimensions dependence of a number of particles; investigation of transverse coupling on the beam dimensions; investigation of beam-beam effects at high luminosity; diagnostic of phase oscillations (fig. 5).

\section{FURTHER DEVELOPMENT OF THE 5 DIAGNOSTICS}

In the immediate future we plan to replace the linear CCD arrays by CCD matrix SONY ICX084AL $(659 \times 489$ pixels). Many of the problems of accelerators physics call for information about turn-to turn dynamic of a beam profile. We plan to employ multi-anode PMT Hamamatsu R5900U-00-L16 for these purposes.

\section{CONCLUSION}

The system of the beam dimensions measurement of the VEPP-4M collider has been in operation for 15 years. A variety of the experiments were performed with the use of this system. The immediate plans to partially up-grade the diagnostic consist of applying modern types of photodetectors.

\section{REFERENCES}

[1] V.V. Anashin et al., Proceedings of $6^{\text {th }}$ Europian Particle Accelerator Conference, EPAC 98, Stockholm, 1998, v.1, p. 400.

[2] V.E. Blinov, A.V. Bogomyakov, S.E. Karnaev, V.A. Kiselev, o. a., Development of resonance depolarization method at VEPP-4M for high presision measurement of tau lepton mass, presented at PAC 01.

[3] E. I. Zinin, PhD Thesis, Budker Institute of Nuclear Physics, Novosibirsk, 1984 (in Russian).

[4] Coppens J., Luijckx G., Zinin E. Commissioning of a bunch length monitor at AmPS // Proceedings of the fifth European particle accelerator conference: EPAC 96, Sitges (Barcelona), 10 - 14 June 1996. - Bristol Philadelphia: Inst. of physics, 1996. - Vol. 2. - p. 1704 - 1706. 